\title{
A GENERAL ANALYSIS OF GENETIC MODELS WITH FREQUENCY-DEPENDENT MATING
}

\author{
P. O'DONALD \\ Department of Genetics, University of Cambridge, Downing Street, Cambridge CB2 3EH, England
}

Received 11.x.79

\section{SuMmary}

\begin{abstract}
A particular phenotype is assumed to mate with a frequency that is any general function, $g(w)$, of its population frequency, $w$. Genetic models are analysed in which two phenotypes, one dominant to the other, mate with relative frequencies $g(w)$ and $1-g(w)$. If the selection that determines the function $g(w)$ acts either on one sex alone or on both sexes equally, then it is shown that the equilibrium frequency, the conditions for the stability of the equilibrium and the eigenvalue that determines the rate of approach to the equilibrium can all be found in terms of simple formulae containing the function $g(w)$ and its derivative. These formulae are applied to some specific models of frequency-dependent sexual and natural selection.
\end{abstract}

\section{INTRODUCTION}

Sexual selection usually entails frequency-dependent mating (O'Donald, $1977 a, b, 1978 a, b, 1979 b)$. In even the simplest models of female preference or male competition, different phenotypes mate with probabilities that are functions of the phenotypes' population frequencies. Suppose, for example, that some females have a fixed preference for one particular male phenotype and other females a fixed preference for another phenotype. The rarer phenotype will tend to be at an advantage over the commoner, since a relatively greater proportion of females will express a preference for the rarer than for the common phenotype. Specifically, if a proportion $\alpha$ prefer phenotype $A$ and $\beta$ prefer phenotype $a$, while the remaining $1-\alpha-\beta$ mate at random, then the total probabilities that individuals who are $A$ or $a$ will find mates are given by

$$
\begin{aligned}
P_{T}(A) & =\alpha+(1-w)(1-\alpha-\beta) \\
P_{T}(a) & =\beta+w(1-\alpha-\beta)
\end{aligned}
$$

where $1-w$ is the population frequency of $A$ and $w$ the population frequency of $a$. This model assumes that matings are polygynous, since males may be chosen to mate both preferentially and randomly. The relative frequencies of the matings are therefore

$$
\begin{aligned}
& P_{T}(A) /(1-w)=\alpha /(1-w)+1-\alpha-\beta \\
& P_{T}(a) / w=\beta / w+1-\alpha-\beta
\end{aligned}
$$

Thus, if $A$ becomes rarer and hence $1-w$ a smaller quantity, the $A$ males take part in a greater proportion of the matings: their selective advantage increases; it is therefore negatively frequency-dependent.

The model with fixed preferences is an extreme one. More realistically, 
the females may mate preferentially depending upon their chances of meeting a male they prefer. This gives rise to the "encounter models" of the expression of mating preference: a female expresses her preference if she meets one of the males she prefers within a certain number of encounters with courting males (O'Donald, $1978 a, b, 1979 a, b$ ). If she has not encountered one of the preferred males during this time, she then mates at random. This pattern of behaviour will follow if the females respond to the courtship of the different males at different thresholds in their level of stimulation: a female with a lower threshold for $A$ males will only mate with an $a$ male after a certain extra number of encounters has raised her level of stimulation to the higher threshold. The probabilities of mating with $A$ and $a$ males are then given by

$$
\begin{aligned}
P_{T}(A) & =\alpha\left(1-w^{n+1}\right)+\beta(1-w)^{m+1}+(1-w)(1-\alpha-\beta) \\
P_{T}(a) & =\alpha w^{n+1}+\beta\left[1-(1-w)^{m+1}\right]+w(1-\alpha-\beta)
\end{aligned}
$$

As the "encounter parameters", $m$ and $n$, are increased without limit, these probabilities become equal to those of the model with fixed preferences. In other models, the probabilities of mating depend on the relative numbers of the $A$ and $a$ males encountered prior to mating (O'Donald, 1979b). In all the models, the probabilities of mating can be described in terms of a function, $g(w)$, of the frequency, $w$, of the $a$ phenotype; so that in general

$$
\begin{aligned}
P_{T}(A) & =g(w) \\
P_{T}(a) & =1-g(w)
\end{aligned}
$$

In those cases in which the phenotypes are determined genetically by dominant and recessive alleles, simple and general results giving the equilibrium frequency and the rate of approach to it can be derived in terms of the function $g(w)$ and its derivative. This analysis can be applied to any specific model of natural or sexual selection in which the function $g(w)$ can be defined. In this paper I analyse three general cases: (i) selection acts only on males; (ii) selection acts equally on both sexes; (iii) selection acts differently on males and females. The results obtained are then applied to some previously analysed models.

\section{Selection of males alone}

For reasons which Darwin (1871) discussed in detail, sexual selection usually acts only on the males. Sometimes the females, rather than the males, appear to have been sexually selected. In birds, the phalaropes (Phalaropodidae) are a good example: the female acquires the more brightly coloured plumage in the breeding season, defends her territory against other females, and courts the males. Although this is an unusual example, the following analysis applies in general to selection acting on females alone as well as to selection acting on males alone.

If $A$ is genetically dominant, consisting of the genotypes $A A$ and $A a$, and $a$ is recessive, consisting of the genotype $a a$, then the matings of $A A$ and $A a$ will always occur in the ratio of their population frequencies. Thus, if $u, v$ and $w$ are the frequencies of $A A, A a$ and $a a$, the genotypes mate with the following probabilities: 


$$
\begin{aligned}
P_{T}(A A) & =u g(w) /(1-w) \\
P_{T}(A a) & =v g(w) /(1-w) \\
P_{T}(a a) & =1-g(w)
\end{aligned}
$$

where $P_{T}(A)=P_{T}(A A)+P_{T}(A a)=g(w)$. The females, not being selected, mate with the unchanged genotypic frequencies $u, v$ and $w$. Matings then take place at frequencies shown in table 1 . These frequencies are derived on the assumption that between males and females the genotypes unite at random. For example, in sexual selection by female choice, the proportion of females with a preference for a particular phenotype is assumed not to be determined by what genotype the females themselves possess: matings do not take place assortatively, as they would do for example, if females who were $a a$ showed a greater preference for a males than females who were $A A$ or $A a$. This assumption is crucial to the general analysis which follows.

\section{TABLE 1}

Frequencies of matings when selection acts only on one sex

\begin{tabular}{ll} 
Mating & \multicolumn{1}{c}{ Frequency } \\
$A A \times A A$ & $u^{2} g(w) /(1-w)$ \\
$A A \times A a$ & $2 u v g(w) /(1-w)$ \\
$A A \times a a$ & $u w g(w) /(1-w)+u-u g(w)$ \\
$A a \times A a$ & $v^{2} g(w) /(1-w)$ \\
$A a \times a a$ & $v w g(w) /(1-w)+v-v g(w)$ \\
$a a \times a a$ & $w-w g(w)$
\end{tabular}

From the frequencies of the matings given in table 1, we obtain the recurrence equations relating the genotypic frequencies from one generation to the next.

$$
\begin{aligned}
u^{\prime} & =p^{2} g /(1-w) \\
v^{\prime} & =(2 p q-p w) g /(1-w)+p(1-g) \\
w^{\prime} & =\left(q^{2}-q w\right) g /(1-w)+q(1-g)
\end{aligned}
$$

In these equations, $p$ is the gene frequency of the $A$ allele: $p=u+\frac{1}{2} v$; $q=1-p ; p^{\prime}=u^{\prime}+\frac{1}{2} v^{\prime}$. Thus we get the recurrence equation for the gene frequency

$$
p^{\prime}=p\left(1-\frac{1}{2} w\right) g /(1-w)+\frac{1}{2} p(1-g)
$$

In the form of a difference equation, this becomes

$$
\Delta p=\frac{1}{2} p(g-1+w) /(1-w)
$$

At equilibrium when $\Delta p=0$, the phenotype $A$ occurs with frequency

$$
1-w_{*}=g\left(w_{*}\right)
$$

Hence to find the equilibrium frequency, we simply equate the population frequency of $A$ to its probability of mating and solve the equation to find $w_{*}$ and $1-w_{*}$.

At the equilibrium, it is immediately apparent that the genotypes occur in the Hardy-Weinberg ratios, $u_{*}=p_{*}{ }^{2}, v_{*}=2 p_{*} q_{*}$ and $w_{*}=q_{*}{ }^{2}$. This fact was noted in all the specific models of sexual selection that had been 
previously analysed (O'Donald, 1977b, 1978a, b, 1979a, b), and shown to be generally true for any sexual selection model (Karlin, 1978).

General formulae can also be found for the stability conditions and the rate at which the frequencies will approach a stable equilibrium. The recurrence equations can be represently succinctly by the pair of equations

$$
\begin{aligned}
p^{\prime} & =p\left(1-\frac{1}{2} w\right) g /(1-w)+\frac{1}{2} p(1-g) \\
w^{\prime} & =q(q-w) g /(1-w)+q(1-g)
\end{aligned}
$$

Then we find the gradient matrix at the equilibrium point, given by

$$
\left[\begin{array}{ll}
\frac{\partial p^{\prime}}{\partial p_{*}} & \frac{\partial p^{\prime}}{\partial w_{*}} \\
\frac{\partial w^{\prime}}{\partial p_{*}} & \frac{\partial w^{\prime}}{\partial w_{*}}
\end{array}\right]
$$

where the symbols $\partial p^{\prime} / \partial p_{*}, \partial p^{\prime} / \partial w_{*}$, etc., are used to indicate that the partial derivatives are to be evaluated at the equilibrium point $p=p_{*}$, $w=w_{*}$. On carrying out this differentiation, we find that the gradient matrix takes the form

where

$$
\left[\begin{array}{cc}
1 & \frac{1}{2} \theta / q_{*} \\
-2 q_{*} & -\theta
\end{array}\right]
$$

$$
\theta=p_{*} q_{*}\left(1+\frac{d g}{d w_{*}}\right) / g\left(w_{*}\right)
$$

Near equilibrium, the equation for the rate of approach to $w_{*}$ is therefore a constant factor times the equation for the rate of approach to $p_{*}$. If we put

$$
\begin{aligned}
& p=p_{*}+x \\
& w=w_{*}+y
\end{aligned}
$$

where $x$ and $y$ are deviations from $p_{*}$ and $w_{*}$, then approximately, near the equilibrium point

showing that

$$
\left[\begin{array}{l}
x^{\prime} \\
y^{\prime}
\end{array}\right]=\left[\begin{array}{cc}
1 & \frac{1}{2} \theta / q_{*} \\
-2 q_{*} & -\theta
\end{array}\right] \cdot\left[\begin{array}{l}
x \\
y
\end{array}\right]
$$

and hence that

$$
y^{\prime}=-2 q_{*} x^{\prime}
$$

$$
x^{\prime}=x(1-\theta)
$$

The quantity $\lambda=1-\theta$ is the non-zero eigenvalue of the gradient matrix. It may also be found using an approximation by complete differentiation of the equation for $p^{\prime}$ or $\Delta p$ (O'Donald, 1979a, 1979b). Differentiating the expression for $\Delta p$, we obtain

$$
\begin{gathered}
\frac{d \Delta p}{d p}=\frac{1}{2}(g-1+w) /(1-w)+\frac{1}{2} p\left[(1-w)\left(\frac{d g}{d w} \cdot \frac{d w}{d p}+\frac{d w}{d p}\right)\right. \\
\left.-\frac{d w}{d p}(g-1+w)\right] /(1-w)^{2}
\end{gathered}
$$


At equilibrium, when $g(w)=1-w$,

$$
\frac{d \Delta p}{d p}=\frac{1}{2} p\left(\frac{d g}{d w_{*}} \cdot \frac{d w}{d p_{*}}+\frac{d w}{d p_{*}}\right) /\left(1-w_{*}\right)
$$

If we now assume that Hardy-Weinberg ratios hold near equilibrium, then

so that

$$
\begin{gathered}
w=q^{2} \\
\frac{d w}{d p}=-2 q
\end{gathered}
$$

and hence

$$
\frac{d \Delta p}{d p}=-p_{*} q_{*}\left(1+\frac{d g}{d w_{*}}\right) / g\left(w_{*}\right)
$$

$$
\begin{aligned}
\lambda & =1+\frac{d \Delta p}{d p_{*}} \\
& =1-\theta
\end{aligned}
$$

The equilibrium point, $w_{*}$, will be stable provided that $\lambda<1$. Since at a polymorphic equilibrium, $p_{*}, q_{*}, g\left(w_{*}\right)>0$, the condition for stability becomes simply

$$
1+\frac{d g}{d w_{*}}>0
$$

The rate of approach can be expressed by the equation

$$
p_{n}-p_{*}=\lambda^{n}\left(p_{o}-p_{*}\right)
$$

The eigenvalue $\lambda$ is thus the multiplying factor that determines the geometric rate of approach of $p_{n}$ to $p_{*}$.

\section{Selegtion of Both SEXes Equally}

If both sexes are subject to the same selection, the genotypes of both sexes take part in matings with probabilities

$$
\begin{aligned}
P_{T}(A A) & =u g(w) /(1-w) \\
P_{T}(A a) & =v g(w) /(1-w) \\
P_{T}(a a) & =1-g(w)
\end{aligned}
$$

In sexual selection, this would imply that females compete to be fertilised just as males compete to fertilise the females. Ladybirds appear to compete in this way. Strong frequency-dependent mating for melanic and nonmelanic ladybirds takes place in both sexes (Muggleton, 1979). The data give a good fit to models of mating preferences (O'Donald and Muggleton, 1979). In ladybirds, females must mate a number of times to fertilise all their eggs, the same female mating successively with a number of different males. This explains why sexual selection acts on both sexes. 
Assuming there is no assortment in the matings between the different genotypes, the recurrence equations are easily shown to be

$$
\begin{aligned}
u^{\prime}= & p^{2} g^{2} /(1-w)^{2} \\
v^{\prime}= & 2 p(q-w) g^{2} /(1-w)^{2}+2 p g(1-g) /(1-w) \\
w^{\prime}= & (q-w)^{2} g^{2} /(1-w)^{2}+2(q-w) g(1-g) /(1-w) \\
& +(1-g)^{2}
\end{aligned}
$$

which can be reduced to the pair of equations

$$
\begin{aligned}
p^{\prime} & =p g /(1-w) \\
w^{\prime} & =(1-w-p g)^{2} /(1-w)^{2} .
\end{aligned}
$$

Thus we obtain the difference equation

$$
\Delta p=p(g-1+w) /(1-w)
$$

which is exactly twice the corresponding difference of the model in which selection acts only on one of the sexes. The equilibrium frequency is thus the same in models of selection acting either on one sex or on both. The $A$ phenotype is at equilibrium when

$$
1-w_{*}=g\left(w_{*}\right)
$$

If we assume that near equilibrium $w=q^{2}$ and $d w / d p=-2 q$, then from the results given in section 2 it follows immediately that

and hence

$$
\frac{d \Delta p}{d p}=-2 p_{*} q_{*}\left(1+\frac{d g}{d w_{*}}\right) / g\left(w_{*}\right)
$$

where, as before

$$
\lambda=1-2 \theta
$$

$$
\theta=p_{*} q_{*}\left(1+\frac{d g}{d w_{*}}\right) / g\left(w_{*}\right)
$$

This is easily proved, since the gradient matrix has values

$$
\left[\begin{array}{cc}
1 & \theta / q_{*} \\
-2 q_{*} & -2 \theta
\end{array}\right]
$$

with a non-zero eigenvalue $\lambda=1-2 \theta$. Thus when selection acts on both sexes, the rate of approach is geometric with multiplying factor

$$
\lambda=1-2 \theta
$$

whereas, when selection acts only on one sex, the corresponding factor is

$$
\lambda=1-\theta
$$

The equilibria are stable under the same conditions in the two cases, but selection is faster when acting on both sexes.

\section{Selection of MALES Different fRom Females}

When selection acts differently on males than on females, analysis of the model no longer gives equations with a general structure similar to that 
when selection acts only on one sex or equally on both. Selection of males and females would be different if the phenotypes $A$ and $a$ were subjected to natural selection in both sexes, but to sexual selection only in males. Natural selection can be measured by a selective coefficient, $t$, giving the relative advantage or disadvantage of one or other of the phenotypes-the recessive $a$ for example. Then, after natural selection has taken place, $A$ and $a$ will occur in the population at frequencies

$$
\begin{aligned}
& 1-h(w)=(1-w) /(1-t w) \\
& h(w)=w(1-t) /(1-t w)
\end{aligned}
$$

The selective coefficient may be constant or frequency-dependent. After sexual selection of the males, the genotypes take part in matings with the following probabilities for males $(m)$ and females $(f)$.

$$
\begin{aligned}
P_{T}(A A)_{m} & =u g(h(w)) /(1-w) \\
P_{T}(A a)_{m} & =v g(h(w)) /(1-w) \\
P_{T}(a a)_{m} & =1-g(h(w)) \\
P_{T}(A A)_{f} & =u(1-h(w)) /(1-w) \\
P_{T}(A a)_{f} & =v(1-h(w)) /(1-w) \\
P_{T}(a a)_{f} & =h(w)
\end{aligned}
$$

The genotypic frequencies in subsequent generations are determined by the recurrence equations

$$
\begin{aligned}
u^{\prime}= & p^{2} g(1-h) /(1-w)^{2} \\
v^{\prime}= & 2 p(q-w) g(1-h) /(1-w)^{2} \\
& +p[g h+(1-g)(1-h)] /(1-w) \\
w^{\prime}= & {[h+(q-w)(1-h) /(1-w)][(1-g)+(q-w) g /(1-w)] }
\end{aligned}
$$

and so for the gene frequency

giving at equilibrium

$$
p^{\prime}=\frac{1}{2} p(1-h+g) /(1-w)
$$

$$
1-w_{*}=\frac{1}{2}\left(1-h_{*}+g_{*}\right)
$$

where $g_{*}=g\left(h\left(w_{*}\right)\right)$ and $h_{*}=h\left(w_{*}\right)$. It is clear that the Hardy-Weinberg ratios do not occur at equilibrium in this model, for we have

$$
u_{*}=4 p_{*}^{2} g_{*}\left(1-h_{*}\right) /\left(1-h_{*}+g_{*}\right)
$$

O'Donald (1978b, 1979b) analysed models in which a character deleterious to survival was preferentially favoured in males. If females have a fixed preference, $\beta$, for the recessive $a$, then with natural selection acting against $a$, the males mate with probabilities

so that

$$
\begin{aligned}
P_{T}(A) & =(1-\beta)(1-w) /(1-t w) \\
P_{T}(a) & =\beta+w(1-\beta)(1-t) /(1-t w)
\end{aligned}
$$

$$
\begin{aligned}
& g(w)=(1-\beta)(1-w) /(1-t w) \\
& h(w)=w(1-t) /(1-t w)
\end{aligned}
$$


and at equilibrium

$$
w_{*}=\frac{1}{2} \beta / t
$$

as previously obtained (O'Donald, 1978b, 1979b). At this value

and therefore

$$
\begin{aligned}
& g_{*}=(1-\beta)\left(t-\frac{1}{2} \beta\right) / t\left(1-\frac{1}{2} \beta\right) \\
& h_{*}=\frac{1}{2} \beta(1-t) / t\left(1-\frac{1}{2} \beta\right)
\end{aligned}
$$

$$
u_{*}=p_{*}{ }^{2}(1-\beta) /\left(1-\frac{1}{2} \beta\right)^{2}
$$

showing that $A A$ is not present at the Hardy-Weinberg frequency $p_{*}{ }^{2}$. However, if natural and sexual selection act only on males, then

$$
\begin{aligned}
w_{*} & =\beta / t \\
p_{*} & =1-\sqrt{\beta / t} \\
u_{*} & =p_{*}{ }^{2}
\end{aligned}
$$

When the Hardy-Weinberg frequencies are not maintained at equilibrium, the gradient matrix no longer conforms to the simple structure found for the general models analysed in sections 2 and 3 . If selection is weak, however, the deviations from Hardy-Weinberg ratios will always be small and the rate of approach to equilibrium can be obtained approximately using the equation

$$
\lambda=1+\frac{d \Delta p}{d p}
$$

\section{Some APplications of the General FormulaE}

The general formulae derived in sections 2 and 3 can be used to find equilibrium frequencies and rates of approach to equilibrium in specific models of selection. The models of sexual selection I shall consider have all been described in detail in Genetic Models of Sexual Selection (O'Donald, 1979b), though some of them were first analysed in earlier papers. I shall only consider those models of sexual selection in which males alone are selected: these are the most realistic models biologically, for sexual selection has usually favoured characters that are phenotypically expressed only in males.

(i) Constant preferential mating of two phenotypes (O'Donald, 1973, $1977 b, 1979 b)$

A proportion $\alpha$ of the females prefer $A$ males and $\beta$ prefer $a$ males; the remaining $1-\alpha-\beta$ mate at random. Then we have

$$
g(w)=\alpha+(1-w)(1-\alpha-\beta)
$$

which is the probability that an $A$ male is chosen for mating. At equilibrium

$$
\begin{aligned}
& 1-w_{*}=\alpha /(\alpha+\beta) \\
& \frac{d g}{d w_{*}}=-(1-\alpha-\beta) \\
& \lambda=1-p_{*} q_{*}(\alpha+\beta)^{2} / \alpha
\end{aligned}
$$


where

$$
\begin{aligned}
& p_{*}=1-\sqrt{\beta /(\alpha+\beta)} \\
& q_{*}=\sqrt{\beta /(\alpha+\beta)}
\end{aligned}
$$

(ii) Sexual and natural selection of a recessive (O'Donald, 1973, $1977 b, 1979 b)$

After natural selection has taken place, males with phenotypes $A$ and $a$ are chosen for mating with population frequencies $(1-w) /(1-t w)$ and $w(1-t) /(1-t w)$. A proportion $\beta$ of the females mate with the $a$ males; $1-\beta$ mate at random. Therefore

$$
\begin{aligned}
& g(w)=(1-\beta)(1-w) /(1-t w) \\
& w_{*}=\beta / t \\
& \frac{d g}{d w_{*}}=-(1-t) /(1-\beta) \\
& \lambda=1-p_{*} q_{*} t /(1-\beta)
\end{aligned}
$$

(iii) "Encounter model" of sexual selection with $\mathrm{n}=\mathrm{m}=1$

(O’Donald, 1978a, 1979b)

The general encounter model is briefly described in the introduction to this paper. When $n=m=1$, females with preferences, who do not meet a male they prefer on their first encounter with a courting male, then mate at random on their second encounter. Putting $n=m=1$ in the expression for $P_{T}(A)$, we find that

$$
\begin{aligned}
g(w) & =\alpha\left(1-w^{2}\right)+\beta(1-w)^{2}+(1-w)(1-\alpha-\beta) \\
& =(1-w)(1+\alpha w-\beta w)
\end{aligned}
$$

If $\alpha>\beta, w_{n} \rightarrow 0:$ if $\beta>\alpha, w_{n} \rightarrow 1$. In the case when $\alpha>\beta$,

$$
\frac{d g}{d w_{*}}=\alpha-\beta-1
$$

Fixation of $A$ takes place at the rate given by

$$
\lambda=1-p_{*} q_{*}(\alpha-\beta)
$$

(iv) Encounter model with $\mathrm{n}=\mathrm{m}=2$ (O'Donald, 1978a, 1979b)

In this model

$$
\begin{aligned}
& g(w)=(1-w)\{1+w[\alpha-2 \beta+w(\alpha+\beta)]\} \\
& w_{*}=(2 \beta-\alpha) /(\alpha+\beta) \\
& \quad \frac{d g}{d w_{*}}=(2 \alpha-\beta)(2 \beta-\alpha) /(\alpha+\beta)-1 \\
& \lambda=1-p_{*} q_{*}(2 \beta-\alpha)
\end{aligned}
$$

This is a new result. It proves, as I had previously found by a different method, that the equilibrium is stable if $2 \beta>\alpha$. 
(v) Encounter model with sexual and natural selection of a recessive (O’Donald, 1978b, 1979b)

If females mate at random after only one encounter $(m=1)$, then $A$ males mate with probability

$$
g(w)=(1-w)[1-\beta w-t w+\beta t w] /(1-t w)^{2}
$$

There is an unstable equilibrium at the point

$$
w_{*}=(t-\beta+\beta t) / t^{2}
$$

If $\beta>t /(1-t), w_{n} \rightarrow 1, g\left(w_{n}\right) \rightarrow 0$; if $\beta<t /(1-t), w_{n} \rightarrow 0, g\left(w_{n}\right) \rightarrow 1$. In the latter case,

$$
\frac{d g}{d w_{*}}=t-\beta+\beta t-1
$$

giving

$$
\lambda=1-p_{*} q_{*}(t-\beta+\beta t)
$$

for the rate of approach to fixation of $A A$.

If females mate at random after two encounters with courting males $(m=2)$, then

$$
g(w)=(1-w)\left[1-2 w(\beta+t-\beta t)+w^{2}\left(\beta+t^{2}-\beta t^{2}\right)\right](1-t w)^{3}
$$

At equilibrium

$$
t^{3} w^{2}+w\left(\beta-2 t^{2}-\beta t^{2}\right)-2 \beta+t+2 \beta t=0
$$

and approximately if $t$ is a small or fairly small quantity

$$
\begin{aligned}
& w_{*}=(2 \beta-t-2 \beta t) /\left(\beta-2 t^{2}-\beta t^{2}\right) \\
& \lambda=1-p_{*} q_{*}\left(\beta-2 t^{2}-\beta t^{2}\right) /\left(1-t w_{*}\right)^{3}
\end{aligned}
$$

(vi) Frequency-dependent natural selection (Clarke and O'Donald, 1964)

Constant selective forces acting on two phenotypes will lead to fixation of the advantageous type. Negative frequency-dependent selection will give rise to stable polymorphism. For example, suppose that the fitnesses of $A$ and $a$ are determined by frequency-dependent selective coefficients, $s(1-w)$ and $t w$ respectively. The fitnesses are then in the ratio $1-s(1-w)$ to $1-t w$ and the two phenotypes mate in the proportions

$$
\frac{(1-w)-s(1-w)^{2}}{1-s(1-w)^{2}-t w^{2}}
$$

and

$$
\frac{w-t w^{2}}{1-s(1-w)^{2}-t w^{2}}
$$

Therefore

$$
g(w)=\frac{(1-w)-s(1-w)^{2}}{1-s(1-w)^{2}-t w^{2}}
$$

At the equilibrium point when

$$
1-w_{*}=g\left(w_{*}\right)
$$


we find that

$$
\begin{gathered}
w_{*}=s /(s+t) \\
\frac{d g}{d w_{*}}=\frac{-1+2 s t /(s+t)}{1-s t /(s+t)} \\
\lambda=1-\frac{2 s(\sqrt{s(s+t)}-s)}{s+t-s t}
\end{gathered}
$$

This result generalises a result previously derived by Clarke and O'Donald (1964). Clarke and O'Donald had assumed that $s=t$, and obtained

$$
\lambda=\frac{1+\frac{1}{2} t-t \sqrt{ } 2}{1-\frac{1}{2} t}
$$

Putting $s=t$ in the general formula gives Clarke and O'Donald's result.

As we have seen, both in general and in specific examples, the equilibrium frequency can always be found by equating the frequency of one of the phenotypes to its probability of mating. This would be the obvious way of finding the equilibrium frequency if reproduction were asexual. The recurrence equation for the frequency of the $a$ phenotype would then simply be

$$
w^{\prime}=1-g(w)
$$

so that we should immediately get the equation for equilibrium

$$
1-w_{*}=g\left(w_{*}\right)
$$

exactly as when $a$ is a recessive that reproduces sexually. If reproduction were asexual, the rate of approach to this equilibrium would be determined by the factor

$$
\lambda=-\frac{d g}{d w_{*}}
$$

For example in the model with fixed mating preferences in which

$$
\begin{gathered}
g(w)=\alpha+(1-w)(1-\alpha-\beta) \\
-\frac{d g}{d w_{*}}=1-\alpha-\beta=\lambda
\end{gathered}
$$

This can be shown directly from the recurrence equation for an asexual species

$$
1-w^{\prime}=\alpha+(1-w)(1-\alpha-\beta)
$$

which can be written in the form

giving

$$
w^{\prime}-w_{*}=(1-\alpha-\beta)\left(w-w_{*}\right)
$$

where, as before,

$$
w_{n}-w_{*}=\lambda^{n}\left(w_{o}-w_{*}\right)
$$

$$
\lambda=1-\alpha-\beta
$$

Under asexual reproduction, the condition for stability,

$$
-\frac{d g}{d w_{*}}<1
$$


or

$$
1+\frac{d g}{d w_{*}}>0
$$

is exactly the same as the condition for stability in the models with sexual reproduction of dominant and recessive phenotypes. Thus if we take the false premise of asexual reproduction, we shall still obtain the correct equilibrium frequency and conditions for stability, but the rate of approach to equilibrium will be wholly erroneous. Since it appears to be the standard procedure of "sociobiologists", in investigating the evolutionary outcomes of different behavioural strategies, to assume, implicitly and falsely, that reproduction is asexual, the general formulae established in this paper may be used to indicate what valid conclusions have been reached by this erroneous procedure and how a specific model might be analysed correctly. Although sociobiological theory often appears to dictate that behavioural models should be described in game-theoretic terms, these models can usually be stated in straightforward, population genetic terms with frequencydependent selection. If the selection is acting on two phenotypes with dominance - the simplest model to start with - then the formulae given here should be applicable. However, if heterozygotes are to some extent separately selected, the evolutionary outcomes may become very complicated, sometimes with a multiplicity of equilibria, some stable and others unstable; and the assumption of asexual reproduction will produce only error.

\section{REFERENCES}

Clarke B., AND o'donald, P. 1964. Frequency-dependent selection. Heredity, 19, 201-206. DARwin, C. 1871. The Descent of Man and Selection in Relation to Sex. John Murray, London. KARLIN, s. 1978. Comparisons of positive assortative mating and sexual selection models. Theor. Pop. Biol., 14, 281-312.

MUGGleton, J. 1979. Non-random mating in wild populations of polymorphic Adalia bipunctata. Heredity, 42, 57-65.

o'DONALD, P. 1973. Models of sexual and natural selection in polygamous species. Heredity, $31,145-156$.

o'DONALD, P. 1977a. Advantage of rare males in models of sexual selection. Nature, 267, 151-154.

o'donald, P. 1977b. Theoretical aspects of sexual selection. Theor. Pop. Biol., 12, 298-334. o'DONALD, P. 1978a. Theoretical aspects of sexual selection: a generalized model of mating behaviour. Theor. Pop. Biol., 13, 226-243.

O'DONALD, P. $1978 b$. A general model of mating behaviour with natural selection a female preference. Heredity, 40, 427-438.

o'DONALD, P. 1979a. Theoretical aspects of sexual selection: variation in threshold of female mating response. Theor. Pop. Biol., 15, 191-204.

o'donald, P. 1979b. Genetic Models of Sexual Selection. Cambridge University Press.

o'DONALD, P., AND MUGGLETON, J. 1979. Melanic polymorphism in ladybirds maintained by sexual selection. Heredity, 43, 143-148. 